\title{
The persistence in real interest rates: Does it solve the intertemporal consumption behavior puzzle?
}

\begin{abstract}
This paper investigates the stationarity behavior of the ex-post real interest rates (RIRs) for 12 Asian countries. Formal tests conducted indicate that high persistence is an intrinsic characteristic in the majority of the RIRs. We consider local-persistent model to assess the degree of persistence in these series. The findings from this devise reveal that RIRs are persistent, but are characterized by a mean-reverting process. The consistency of the persistence comparable amongst the series is confirmed after accounting for the volatility of the consumption growth. Applying a test proposed by Leybourne et al. (2007a) that allows for long memory dynamics, we reconfirm the characteristic of the series. Building on previous studies, this paper provides favorable support for the long-run Fisher hypothesis and help to solve an intertemporal consumption behavior puzzle across the emerging and advanced countries. Finally, the results for the G-7 countries are presented for comparison.
\end{abstract}

Keyword: Real interest rates; Consumption growth; Half-life; Asian countries 